\title{
Immune-modulating effects in mouse dendritic cells of lactobacilli and bifidobacteria isolated from individuals following omnivorous, vegetarian and vegan diets
}

Diomira Luongo ${ }^{\mathrm{a}}$, Lucia Treppiccione ${ }^{\mathrm{a}}$, Alida Sorrentino ${ }^{\mathrm{a}}$, Ilario Ferrocino ${ }^{\mathrm{b}}$, Silvia Turroni ${ }^{\mathrm{c}}$, Monica Gatti ${ }^{\mathrm{d}}$, Raffaella Di Cagno ${ }^{\mathrm{e}}$, Yolanda Sanz ${ }^{\mathrm{f}}$, Mauro Rossi ${ }^{\mathrm{a} *}$.

${ }^{\text {a }}$ Institute of Food Sciences, CNR, Avellino, Italy

${ }^{\mathrm{b}}$ Department of Agricultural, Forest and Food Science, University of Turin, Grugliasco, Italy

${ }^{c}$ Department of Pharmacy and Biotechnology, Alma Mater Studiorum University of Bologna, Bologna, Italy

${ }^{\mathrm{d}}$ Laboratory of Food Microbiology, Department of Food Science, University of Parma, Parco Area delle Scienze 48/A, Parma, Italy

${ }^{\mathrm{e}}$ Department of Soil, Plant and Food Sciences, University of Bari Aldo Moro, Bari, Italy

${ }^{\mathrm{f}}$ Microbial Ecology, Nutrition and Health Research Unit, Institute of Agrochemistry and Food Technology, National Research Council (IATA-CSIC), Valencia, Spain.

*Correspondence to: Dr. Mauro Rossi, Istituto di Scienze dell'Alimentazione-CNR, via Roma 64, 83100 Avellino. Tel/fax: \#39 0825299308; e-mail mrossi@isa.cnr.it

Abbreviations used in this paper: DCs, dendritic cells; TPY, trypticase-phytone-yeast extract; MRS, de Man, Rogosa and Sharpe medium; GM-CSF, granulocyte-macrophage colony-stimulating factor; SCFAs, short-chain fatty acids; LAB, lactic acid bacteria; CTLA-4, cytotoxic T-lymphocyte antigen 4 . 


\begin{abstract}
Lactobacilli and bifidobacteria play a primary role in modulation of gut immunity. By considering that microbiota composition depends on various factors, including diet, we asked whether functional differences could characterize faecal populations of lactobacilli and bifidobacteria isolated from individuals with different dietary habits. 155 healthy volunteers who followed omnivorous, ovo-lacto-vegetarian or vegan diets were recruited at four Italian centres (Turin, Parma, Bologna and Bari). Faecal samples were collected; lactobacilli and bifidobacteria were isolated on selective media and their immunomodulatory activity was tested in mouse dendritic cells (DCs). Pre-incubation with lactobacilli increased LPS-induced expression of the maturation markers CD80 and CD86, whereas pre-incubation with bifidobacteria decreased such expression. Analysis of the cytokine profile indicated that strains of both genera induced down-regulation of IL12 and up-regulation of IL-10, whereas expression of TNF- $\alpha$ was not modulated. Notably, analysis of anti-inflammatory potential (IL-10/IL-12 ratio) showed that lactobacilli evoked a greater antiinflammatory effect than did bifidobacteria in the omnivorous group $(P<0.05)$. We also found significantly reduced anti-inflammatory potential in the bacterial strains isolated from Bari's volunteers in comparison with those from the cognate groups from the other centres. In conclusion, lactobacilli and bifidobacteria showed a genus-specific ability of modulating in vitro innate immunity associated with a specific dietary habit. Furthermore, the geographical area had a significant impact on the anti-inflammatory potential of some components of faecal microbiota.
\end{abstract}

Keywords: omnivorous diet, vegetarian diet, vegan diet, microbiota, immunity, dendritic cells. 


\section{Introduction}

Several endogenous and environmental factors can impact both the composition and functionality of gut microbiota throughout life [1]. In particular, substantial evidence indicates that diet is instrumental in the structure and functionality of gut microbiota [2-6]. Epidemiologic evidence also suggests that modulation of immune response mechanisms in the gut can contribute to the development of gastrointestinal disorders and allergic diseases. In this context, lactobacilli and bifidobacteria are considered key players because they constitute important members of the normal intestinal microbiota in animals and humans, particularly bifidobacteria in infants [7,8 ]. The current knowledge of mechanisms by which intestinal microbiota influences both regulatory and effector components of the immune system highlights the importance of enterocyte and dendritic cell (DC) interactions. DCs are considered to be gate-keepers of the immune system, and their contact with microbiota is crucial for proper development of gut immunity [9]. This contact is essentially achieved by interacting directly with bacteria that have gained access to lymph nodes and Peyer's patches via M cells. Furthermore, subepithelial DCs may sample the microbiota by passing their dendrites between epithelial cells into the gut lumen [10]. However, the precise mechanisms by which intestinal microbiota can influence the development and function of DCs remain to be further elucidated. Additionally, the composition of a healthy microbiota, which is required to confer immune protection, is not known, nor is the influence of dietary habits to improve the function of the microbiota. Herein, we addressed these issues by establishing whether lactobacilli and bifidobacteria species and strains arising from specific diets actually translate to the modulation of immune function. We evaluated, for the first time, a large microbiological screening of lactobacilli and bifidobacteria isolated from individuals undergoing omnivorous, vegan and ovo-lactovegetarian diets $[11,12]$ to determine their role in modulating in vitro immune markers of mouse dendritic cell functions. Our data revealed genus-specific effects of selected bacteria on the maturation of DCs, as well as on inducible immune mediators produced by DCs in vitro, thus establishing a differential degree of modulation of the phenotype of DCs. Importantly, the latter parameter was found to be dependent on dietary habit. 


\section{Materials and Methods}

\subsection{Participant recruitment and faecal sample collection}

Healthy adult volunteers $(\mathrm{n}=155)$ who followed an omnivorous $(\mathrm{n}=55)$, ovo-lacto-vegetarian $(\mathrm{n}=$ $53)$ or vegan $(n=47)$ diet for more than 1 year were recruited at 4 Italian centres (Bari, Bologna, Parma and Turin) [11-13] (https://clinicaltrials.gov; ClinicalTrials.gov Identifier: NCT02118857; MRMOVVD), as indicated in Table 1. The research was conducted according to the Declaration of Helsinki. Informed consent was obtained from all subjects. The compliance to the declared diet type was verified by means of a 7-day weighed food diary, completed every day for a total of 7 days [11]. Three faecal samples/volunteer (ca. $15 \mathrm{~g}$ ) were collected for 3 consecutive weeks (once per week) at home, transferred to sterile tubes containing $10 \mathrm{ml}$ of liquid Amies transport medium (Oxoid, Milan, Italy) and stored at $4^{\circ} \mathrm{C}$. The specimens were then transported to the laboratory within 12 hours and immediately processed.

\subsection{Isolation and growth of lactobacilli and bifidobacteria}

$10 \mathrm{~g}$ of faeces from each volunteer was homogenized with $90 \mathrm{ml}$ of Ringer's solution (Oxoid) for 2 min in a stomacher (LAB Blender 400, PBI, Italy) at room temperature. Serial dilutions were prepared in Ringer's solution, and $100 \mu \mathrm{l}$ aliquots of each dilution were included into Rogosa Agar (Oxoid) with $21 \mu \mathrm{M}$ acetic acid or spread onto Bifidobacterium Agar (Becton Dickinson, Milan, Italy) [12-14]. Growth conditions were aerobic at $30^{\circ} \mathrm{C}$ for $48 \mathrm{~h}$ and anaerobic at $37^{\circ} \mathrm{C}$ for $48-72 \mathrm{~h}$ for selection of mesophilic lactobacilli and bifidobacteria, respectively. For each faecal sample, 10 random colonies were picked from appropriate plate dilutions for analysis in vitro $[13,15,16]$. Cell morphology and cell motility of selected colonies were evaluated for genus confirmation [17]. Bifidobacteria were cultured in trypticase-phytone-yeast extract (TPY; Oxoid) at $37^{\circ} \mathrm{C}$ under anaerobic conditions and lactobacilli in de Man, Rogosa and Sharpe medium (MRS; Oxoid) at $30^{\circ} \mathrm{C}$ under aerobic conditions; cells were collected during exponential growth phase. Randomly selected cultures were finally checked by PCR-DGGE and sequencing of amplimers [13]. Cell concentration was evaluated by measuring optical density at $600 \mathrm{~nm}$ and converting this value to the corresponding $\mathrm{CFU} \mathrm{m}{ }^{-1}$ value. Bacteria were irradiated with $2800 \mathrm{~Gy}$ (Gray) of $\gamma$-irradiation by Gamma-Cell 1000 (MDS Nordion, Canada) to prevent their proliferation before being used as stimuli for DCs.

\subsection{Mice}


$\mathrm{BALB} / \mathrm{c}$ mice were maintained under pathogen-free conditions at the animal facility of the Institute of Food Sciences. Mice were used at 6-12 weeks of age and were euthanized by inhalation of anaesthesia with isoflurane. These studies were approved by the National Institutional Review Committee.

\subsection{Isolation of bone marrow-derived dendritic cells}

Murine DCs were generated according to a previously published method [18]. In brief, bone marrow cells from the femurs and tibiae of mice were flushed, and bone marrow cell aliquots ( $2 \mathrm{x}$ $10^{6}$ ) were diluted in $10 \mathrm{ml}$ of RPMI 1640 medium supplemented with $25 \mathrm{mM}$ HEPES, antibiotics (penicillin $100 \mathrm{IU} \mathrm{ml}^{-1}$, streptomycin $100 \mathrm{IU} \mathrm{ml}^{-1}$ ), $10 \%$ foetal calf serum and $20 \mathrm{ng} \mathrm{ml}^{-1}$ granulocyte-macrophage colony-stimulating factor (GM-CSF) (culture medium) before being seeded in 100-mm petri dishes (Falcon, Heidelberg, Germany). On day 3, $10 \mathrm{ml}$ of culture medium was added and on day $7,10 \mathrm{ml}$ of the culture medium was replaced with freshly prepared medium. On day 9, non-adherent DCs were harvested by gentle pipetting. Cell aliquots $\left(1 \times 10^{6} \mathrm{ml}^{-1}\right)$ were then placed in 24-well plates and incubated in culture medium with $5 \mathrm{ng} \mathrm{ml}^{-1}$ GM-CSF. Cell viability was microscopically evaluated by dye-exclusion test using Nigrosin (1\% solution) and > $90 \%$ live cells were found in all experiments.

\subsection{Microbial challenge}

DCs $\left(1 \times 10^{6} \mathrm{ml}^{-1}\right)$ were incubated for $24 \mathrm{~h}$ with irradiated bacteria resuspended in complete RPMI medium at a 30:1 bacteria (CFU):DC (n) ratio. Following incubation, cells were treated with $1 \mu \mathrm{g}$ $\mathrm{ml}^{-1}$ LPS for $6 \mathrm{~h}$ (LPS pulse) to induce the maturation of DCs and cultured for $0-24 \mathrm{~h}$ in complete RPMI medium. Cells were collected for RNA analysis. Spent media was centrifuged at 10,000 x g for 10 min to eliminate any residual cells and cell debris, and supernatants were stored at $-80^{\circ} \mathrm{C}$. No $\mathrm{pH}$ change occurred in the medium after $24 \mathrm{~h}$ of bacterial incubation.

\subsection{FACS analysis}

DCs were stained with phycoerythrin (PE)- or fluorescein isothiocyanate (FITC)-conjugated Abs (BioLegend, San Diego, CA, USA) against CD11b, CD11c, CD80 and CD86. Cell staining was analysed using a CyFlow Space flow cytometer (Partec, Munster, Germany) and FlowJo software (Tree Star Inc., Ashland, OR, USA). For each Ab, an isotype control of the appropriate subclass was used.

\subsection{Analysis of cytokine production}


Total RNA was extracted from DCs using the TRIzol Reagent (Life Technologies Italia, Monza $\mathrm{MB}$, Italy) according to the manufacturer's instructions and quantified by fluorimetry using the RiboGreen RNA Reagent (Invitrogen Corp., Carlsbad, CA, USA), and RNA quality was verified by denaturing gel electrophoresis. cDNA was prepared from $1 \mu \mathrm{g}$ of total RNA by reverse transcription with M-MLV Reverse Transcriptase (Invitrogen) and Oligo-(dT) ${ }_{12-18}$ Primer at $42^{\circ} \mathrm{C}$ for 60 min. Real-time PCR was performed using the iCycler $\mathrm{iQ}^{\mathrm{TM}}$ Real-Time Detection System (Bio-Rad, Hercules, CA, USA). Amplification was conducted in a total volume of $25 \mu 1$, containing $\mathrm{iQ}^{\mathrm{TM}}$ SYBR Green Supermix (Bio-Rad), $0.2 \mu \mathrm{M}$ of each primer and cDNA. The reaction conditions for 39 cycles were $95^{\circ} \mathrm{C}$ for $30 \mathrm{~s}, 54.6^{\circ} \mathrm{C}(\mathrm{L}-32), 60^{\circ} \mathrm{C}$ (IL-12, TNF- $\alpha$ ), or $66.0^{\circ} \mathrm{C}$ (IL-10) for $30 \mathrm{~s}$, and $72{ }^{\circ} \mathrm{C}$ for $40 \mathrm{~s}$. The relative gene expression levels of TNF- $\alpha$, IL-12p40 and IL-10 were calculated using the $\Delta \Delta \mathrm{C}_{\mathrm{T}}$ method and presented as fold change in gene expression after normalization to the L-32 housekeeping gene. The following primer sequences were designed and used in this study: L32, forward 5'-AGCAGAGCTGGAGTCGCTTT-3', 5'GGAGCTGCCATCCAAAAGATACTA-3'; TNF- $\alpha$, forward 5'CATCTTCTCAAAATTCGAGTGACAA-3', reverse 5'-TGGGAGTAGACAAGGTACAACCC-3'; IL12p40, forward 5'-GGAAGCACGGCAGCAGAATA-3', reverse 5'AACTTGAGGGAGAAGTAGGAATGG-3'; IL-10, forward 5'GACAATAACTGCACCCACTTC-3', reverse 5'-AGCTGGTCCTTTGTTTGAAAGAAA-3'.

Supernatants from DC cultures were analysed for IL-12, TNF- $\alpha$ and IL-10 protein levels by sandwich-type ELISA. First, $100 \mu$ of capture antibody solution (BioLegend) was dispensed into each well of a 96-well plate (Nunc Maxisorb; eBioscience Inc., San Diego, CA) and incubated overnight at $4^{\circ} \mathrm{C}$. After removal of the capture antibody solution, $100 \mu \mathrm{l}$ of PBS supplemented with $2 \%$ BSA (blocking buffer) was added to each well and incubated at room temperature for $2 \mathrm{~h}$. Next, cytokine standards and samples diluted in blocking buffer supplemented with $0.05 \%$ Tween-20 were added to the respective wells and incubated overnight at $4^{\circ} \mathrm{C}$. After incubation, three washing steps with PBS supplemented with $0.05 \%$ Tween-20 were performed, and $100 \mu$ of biotinylated antibody solution was added to the wells and incubated for $2 \mathrm{~h}$ at room temperature. After three washes, streptavidin-horseradish peroxidase conjugate (1:2000 dilution; BioLegend) was added to the wells and incubated for $1 \mathrm{~h}$ at room temperature. Finally, after washing, $100 \mu \mathrm{l}$ of $63 \mathrm{mM}$ $\mathrm{Na}_{2} \mathrm{HPO}_{4}, 29 \mathrm{mM}$ citric acid ( $\mathrm{pH}$ 6.0) containing $0.66 \mathrm{mg} \mathrm{ml}^{-1}$ o-phenylenediamine/ $\mathrm{HCl}$ and $0.05 \%$ hydrogen peroxide were dispensed into each well, and the wells were allowed to develop. The absorbance was read at $415 \mathrm{~nm}$ and the cytokine concentrations were calculated using standard curves and expressed as $\mathrm{pg} \mathrm{ml}^{-1}$. 


\subsection{Statistical analysis}

Statistical significance was determined by the Kruskal-Wallis test and Dunn's post-hoc test analysis using GraphPad PRISM 4.0 software (GraphPad Software, Inc., La Jolla, CA). A P-value of 0.05 or less was considered to be significant. 


\section{Results}

\subsection{Modulation of maturation markers by diet-selected lactobacilli and bifidobacteria}

To preliminarily examine the effects of bacterial pre-incubation on DC maturation, lactobacilli and bifidobacteria isolated from individuals undergoing different diet regimens were evaluated (lactobacilli from subjects n. 3, 18 and 42; bifidobacteria from subjects n. 14, 21 and 39 of Table 1). DCs were mainly $\mathrm{CD} 11 \mathrm{~b}^{-} \mathrm{CD} 11 \mathrm{c}^{+}$(data not shown) with low expression of the co-stimulatory molecules CD80 and CD86 (Fig. 1, iDCs). Incubation with the TLR4 ligand LPS for 6 hours resulted in increased expression of CD80 and CD86 surface maturation markers, although to different degrees in each independent experiment, as a consequence of heterogeneity in the iDC population (Fig. 1, mDCs). Pre-incubation with lactobacilli further increased the number of $\mathrm{CD}^{+} 0^{+} \mathrm{CD} 86^{+}$cells (Fig. 1, subjects n. 3, 18 and 42). On the contrary, pre-treatment with all three bifidobacteria isolates caused a reduction in LPS-inducible expression of CD80 and CD86 (Fig. 1, subjects n. 14, 21 and 39). Next, we analysed the whole bacteria collection by expressing the number of double positive cells in terms of $\%$ of the positive control (mDCs). The results shown in Fig. 2 indicated that the response varied widely both for lactobacilli (Fig. 2a) and bifidobacteria (Fig. 2b). Nevertheless, it was statistically confirmed that pre-treatment with lactobacilli mainly increased the LPS-induced expression of maturation markers, whereas pre-incubation with bifidobacteria decreased such expression. In addition, we reported that when increases of CD80 and CD86 were induced by bifidobacteria isolates, they never reached the higher levels generated by lactobacilli (Fig. 2a, b). Importantly, the examined diet regimens were not translated into changes in modulatory activity of either lactobacilli or bifidobacteria. Furthermore, no differences were found by examining these data on the basis of their geographical origin (data not shown).

\subsection{Setup of the cytokine analysis in vitro}

Real-time PCR reactions were performed under gene-specific conditions to amplify IL-12p40, IL10 and TNF- $\alpha$ cDNAs, with L-32 as a housekeeping gene. Kinetic analysis of cytokine transcription induced by a LPS pulse in DCs showed that in this model, the examined transcripts promptly peaked at the end of the pulse (IL-12 and TNF- $\alpha$ ) or $1 \mathrm{~h}$ later (IL-10); then, they were rapidly down-regulated following removal of the mitogen (Fig. 3a). Therefore, cytokine mRNA levels were definitively assessed $1 \mathrm{~h}$ after the LPS pulse and compared to cognate protein levels, which were analysed in the supernatants of parallel cultures $24 \mathrm{~h}$ later. To preliminarily estimate the effects of bacterial pre-incubation of iDCs on cytokine expression, we tested lactobacilli isolates collected for three consecutive weeks from subjects n. 3, 18 and 42, who were subjected to different 
diet regimens. IL-12 mRNA levels were differentially regulated by the bacterial samples, but the final outcome was suppression of protein expression in all cases (Fig. 3b, upper panel), suggesting stringent post-transcriptional control of this cytokine by lactobacilli. On the contrary, IL-10 was significantly up-regulated by all samples at the transcriptional level (Fig. 3b, middle panel). TNF- $\alpha$ mRNA was significantly down-regulated even when basal expression of the protein was generally unaffected by lactobacilli challenge (Fig. 3b, bottom panel). By comparing the samples collected in different weeks, we could detect quantitative differences in regulation only for IL-10.

\subsection{Modulation of the cytokine profile by diet-selected lactobacilli and bifidobacteria}

On the basis of the setting up analysis, bacterial collections were screened by determining cytokine protein levels, and data were expressed as \% of the positive control (mDCs). Then, results from each independent experiment were pooled and reported in Fig. 4 and Fig. 5. It was confirmed that maturation of iDCs caused significantly increased expression of IL-12 (Fig. 4a, b) and TNF- $\alpha$ (Fig. $5 \mathrm{a}, \mathrm{b})$. On the contrary, analysis of regulatory IL-10 in a larger sample size indicated that an increase in cytokine protein levels was not consistently obtained by LPS pulse (Fig. 4c, d), reflecting a higher sensitivity of this cytokine to cell heterogeneity of iDCs in different experiments. Pre-incubation with both lactobacilli and bifidobacteria collections generated a wide range of values in the cytokine response. Nevertheless, we found that both genera induced a statistically relevant down-regulation of IL-12 expression that resulted independently of the dietary regimen (Fig. 4a, b). On the other hand, pre-incubation with both lactobacilli and bifidobacteria isolates significantly upregulated IL-10 expression, once again independently of diet (Fig. 4c, d). Yet, expression of the pleiotropic cytokine TNF- $\alpha$ was not significantly modulated by pre-incubation with lactobacilli (Fig. 5a) or bifidobacteria (Fig. 5b). We have shown that, although statistically relevant, both the IL-12 and IL-10 responses varied widely; therefore, to more stringently analyse the impact of dietary regimen, we calculated anti-inflammatory potential in terms of the IL-10/IL-12 ratio from each microbial sample. The results reported in Fig. 6 indicated that most of the examined samples had a ratio $>1$, thus confirming the overall anti-inflammatory activity for both lactobacilli and bifidobacteria. Importantly, a statistically significant higher anti-inflammatory potential was observed for lactobacilli (median >10) in comparison with bifidobacteria in the omnivorous group. Interestingly, by further examining these data on the basis of geographical origin, we found reduced IL-10/IL-12 ratios in the isolates collected from Bari in comparison with the cognate groups from all other centres (Fig. 7a, b); specifically, the Dunn's post-hoc test analysis revealed significant differences between isolates from the vegan group recruited in Turin and those from the vegetarian 
group in Bari among lactobacilli (Fig. 7a) and between isolates from omnivores recruited in Turin and Bari among bifidobacteria (Fig. 7b). 


\section{Discussion}

In this study, we found a genus-specific ability to modulate in vitro mechanisms of innate immunity by faecal populations of lactobacilli and bifidobacteria that was also influenced by the dietary habit. Gut microbiota shapes host immunity by inducing maturation of the gastrointestinal lymphoid tissue. In addition, other mediators, such as polysaccharide A and short-chain fatty acids (SCFAs), have been shown to stimulate Treg-cell differentiation. In particular, SCFAs can induce IL-10 and retinoic acid from DCs, which play a major role in mucosal tolerance [19]. Interestingly, a significant association between consumption of agrarian-based diets and increased levels of faecal SCFAs has been previously found in the individuals herein examined [11]. Furthermore, the culturable lactic acid bacteria (LAB) loads were found to be lower in the vegan and ovo-lactovegetarian groups [12]. On the contrary, no significant difference between the ovo-lacto-vegetarian and omnivorous groups was observed in relation to the culturable bifidobacteria load, although such load was significantly lower in the vegan group [12]. It is well known that the composition and density of microbiota fluctuates with age and can also be influenced by various factors, including diet. Nevertheless, by two years of age, the gut microbiota is essentially dominated by members belonging to the Firmicutes and Bacteroidetes phyla and begins to resemble an adult microbiota [20]. Among the factors, diet clearly influences the developing gut microbiota: after the introduction of solid food, the diversification of Bacteroides spp. and Clostridium spp. rapidly increases, whereas the proportion of Bifidobacterium spp. stabilizes [20]. Lactobacilli numbers found in stool samples seem to be more specifically related to the ingestion of fermented foods. Importantly, strong evidence suggests a primary role of both lactobacilli and bifidobacteria in modulation of immune mechanisms in the gut. Therefore, we addressed this issue by evaluating whether, beyond differences in numbers and species [12], genus-specific functional differences could characterize these bacteria isolated from the screened groups. For each faecal sample, 10 random colonies were picked for analysis from adequate plate dilutions. It has been reported that 10 colonies provide an adequate representation of the major bacterial strains cultured on a selective medium $[15,16]$. We found that immunological outcomes widely varied within each group, suggesting that no bias was introduced by the strategy of bacteria isolation that we adopted. Nevertheless, due to the large number of individuals included in the examined groups, most data were found to be statistically significant. Specifically, we showed that bacteria belonging to the Lactobacillus genus further increased the number of $\mathrm{CD} 80^{+} \mathrm{CD} 86^{+} \mathrm{mDCs}$, whereas this number was decreased by bifidobacteria. To the best of our knowledge, this is the first work showing that the two genera may differentially alter the level of DC activation. Both CD80 and CD86 bind to CD28 
and, with a lower affinity, to its homolog CTLA-4 (cytotoxic T-lymphocyte antigen 4) on T cells. CD28-CD80/86 ligation enhances T-cell proliferation, whereas CTLA-4-CD80/86 interaction inhibits T-cell responses [21]. Consequently, we speculated that the reduced expression of CD80 and CD86 mediated by most of the screened bifidobacteria favours the latter interaction, and this could play a role in maintaining immune tolerance through interaction with CTLA-4. Clearly, the increased number of $\mathrm{CD} 80^{+} \mathrm{CD} 86^{+} \mathrm{mDCs}$ generated by lactobacilli tend to preclude a crucial role for CTLA-4 in lactobacilli-mediated immune homeostasis in the gut. The results of cytokine analysis showed that, independently of the dietary regimen, members of both genera significantly down-regulated the pro-inflammatory cytokine IL-12 and up-regulated the regulatory cytokine IL10 , whereas the expression of the multifunctional cytokine TNF- $\alpha$ was not significantly modulated. Interestingly, experiments showed a more heterogeneous influence of both genera on IL-12 production and CD80+CD86+ surface expression than on IL-10 secretion. These results suggested the existence of strain-specific effects that act on DC mechanisms that make them competent to prime a Th1 response. These results were also in agreement with previous data highlighting substantial differences among lactobacillus strains in the capacity to induce IL-12 and TNF- $\alpha$ production in DC [22]. Notably, the evaluation of anti-inflammatory potential, expressed in terms of the IL-10/IL-12 ratio generated from each sample, showed higher values for lactobacilli than for bifidobacteria in the omnivorous group. Taken together, they are suggestive of genus-specific mechanisms involved in DC education. A possible outcome of this differential activity could be the recruitment of different regulatory cells. Interestingly, oral administration of $B$. breve, but not $L$. casei, in mice has recently been found to induce IL-10-producing $\mathrm{CD}^{+} \mathrm{T}$ cells that possessed properties of $\operatorname{Tr} 1$ cells [23], suggesting that a specific bifidobacterium strain could maintain intestinal homeostasis through the induction of intestinal $\operatorname{Tr} 1$ cells, differently from lactobacilli. Further studies are then required to determine if this property could be considered genus-specific.

It is known that long-term dietary intake influences the structure and functionality of gut microbiota in humans [24]. In the populations examined herein, in agreement with differences in counts for $\mathrm{LAB}$ and bifidobacteria [12], the long-term dietary regimens did generate differences in characterized immune features in the studied isolates. Furthermore, we found reduced antiinflammatory potential in the isolates collected in Bari in comparison with those from the groups at other centres. This finding was well in line with our previous results obtained in Caco-2 cells: lactobacilli collected from the vegetarian group recruited in Bari induced a significant increase in IL-8 expression [13]. By considering sample homogeneity for age, BMI and sex ratio at the different recruitment centres [11], genetic or unselected environmental factors could still influence how and from where a gut microbiota/microbiome is acquired. Interestingly, the significant 
differences between bifidobacteria isolates from omnivores recruited in Turin and Bari tended to exclude a role of diet for this specific feature.

In conclusion, by examining the faecal lactobacilli and bifidobacteria isolates in groups with large sample sizes, we highlighted for the first time the existence of genus-specific differences in their immune-modulatory activity on DCs. In agreement with diet-related variations in plate counts of culturable LAB and bifidobacteria, we also found an association between crucial immune features of bacterial populations and a specific dietary habit. Finally, the geographical area had a significant impact on the anti-inflammatory potential of members of these bacterial genera. 


\section{Acknowledgments}

This study was supported by the Italian Ministry of University and Research (MIUR) PRIN 20102011 program (grant number 2010WZ2NJN; http://www.diet4microgut.it). The funders had no role in the design, analysis or writing of this article. We are grateful to Marco Gobbetti for the coordination of the diet4microgut project. Y. S. was supported by grant AGL2014-52101-P from the Spanish Ministry of Economy and Competitiveness (MINECO).

\section{Conflict of interest}

The authors have no financial or personal conflicts of interest. 


\section{References}

1. M. Candela, E. Biagi, S. Turroni, S. Maccaferri, P. Figini, P. Brigidi, Dynamic efficiency of the human intestinal microbiota, Crit. Rev. Microbiol. 41 (2015) 165-171.

2. B.D. Muegge, J. Kuczynski, D. Knights, J.C. Clemente, A. González, L. Fontana, B. Henrissat, R. Knight, J.I. Gordon, Diet drives convergence in gut microbiome functions across mammalian phylogeny and within humans. Science 332 (2011) 970-974.

3. C. De Filippo, D. Cavalieri, M. Di Paola, M. Ramazzotti, J.B. Poullet, S. Massart, S. Collini, G. Pieraccini, P. Lionetti, Impact of diet in shaping gut microbiota revealed by a comparative study in children from Europe and rural Africa, Proc. Natl. Acad. Sci. USA 107 (2010) 14691-14696.

4. E.F. Murphy, P.D. Cotter, S. Healy, T. M. Marques, O. O'Sullivan, F. Fouhy, S.F. Clarke, P.W. O'Toole, E.M: Quigley, C. Stanton, P.R. Ross, R.M: O'Doherty, F. Shanahan, Composition and energy harvesting capacity of the gut microbiota: relationship to diet, obesity and time in mouse models. Gut 59 (2010) 1635-1642.

5. J. Zimmer, B. Lange, J.S. Frick, H. Sauer, K. Zimmermann, A. Schwiertz, K. Rusch, S. Klosterhalfen, P. Enck, A vegan or vegetarian diet substantially alters the human colonic faecal microbiota, Eur. J. Clin. Nutr. 66 (2012) 53-60.

6. L.A. David, C.F. Maurice, R.N. Carmody, D.B. Gootenberg, J.E. Button, B.E. Wolfe, A.V. Ling, A.S. Devlin, Y. Varma, M.A. Fischbach, S.B: Biddinger, R.J. Dutton, P.J. Turnbaugh, Diet rapidly and reproducibly alters the human gut microbiome, Nature 505 (2014) 559-563.

7. G. Vitaliti, P. Pavone, F. Guglielmo, G. Spataro, R. Falsaperla, The immunomodulatory effect of probiotics beyond atopy: an update, J. Asthma 51 (2014) 320-332.

8. Y. Ringel, E.M.M. Quigley, H.C. Lin, Using Probiotics in Gastrointestinal Disorders, Am. J. Gastroenterol. Suppl 1 (2012) 34-40.

9. K.L. Flannigan, D. Geem, A. Harusato, T.L. Denning, Intestinal Antigen-Presenting Cells: Key Regulators of Immune Homeostasis and Inflammation, Am. J. Pathol. 185 (2015) 1809-1819.

10. M. Rescigno, Dendritic cell-epithelial cell crosstalk in the gut, Immunol. Rev. 260 (2014) 118128.

11. F. De Filippis, N. Pellegrini, L. Vannini, I.B. Jeffery, A. La Storia, L. Laghi, D.I. Serrazanetti, R. Di Cagno, I. Ferrocino, C. Lazzi, S. Turroni, L. Cocolin, P. Brigidi, E. Neviani, M. Gobbetti, P.W. O'Toole, D. Ercolini, High-level adherence to a Mediterranean diet beneficially impacts the gut microbiota and associated metabolome, Gut 2015;pii: gutjnl-2015-309957.

12. I. Ferrocino, R. Di Cagno, M. De Angelis, S. Turroni, L. Vannini, E. Bancalari, K. Rantsiou, G. Cardinali, E. Neviani, L. Cocolin, Fecal microbiota in healthy subjects following omnivore, 
vegetarian and vegan diets: culturable populations and rRNA DGGE profiling, PLoS One 10(6) (2015) e 0128669.

13. D. Luongo, A. Coppola, L. Treppiccione, P. Bergamo, A. Sorrentino, I. Ferrocino, S. Turroni, E. Neviani, R. Di Cagno, L. Cocolin, M. Rossi. Modulation of the cytokine profile in Caco-2 cells by faecal lactobacilli and bifidobacteria from individuals with distinct dietary habits. Cytokine. 90 (2016) 80-87.

14. H. Beerens, An elective and selective isolation medium for Bifidobacterium spp. Lett. Appl. Microbiol. 11 (1990) 155-157.

15. C.L. Hartley, H.M. Clements, K.B. Linton, Escherichia coli in the faecal flora of man, J. Appl. Bacteriol. 43 (1977) 261-269.

16. A.L. McCartney, W. Wenzhi, G.W. Tannock, Molecular analysis of the composition of the bifidobacterial and lactobacillus microflora of humans, Appl. Environ. Microbiol. 62 (1996) 46084613.

17. R. Hartemink, F.M. Rombouts, Comparison of media for the detection of bifidobacteria, lactobacilli and total anaerobes from faecal samples, J. Microbiol. Meth. 36 (1999) 181-192.

18. M.B. Lutz, N. Kukutsch, A.L. Ogilvie, S. Rössner, F. Koch, N. Romani, G. Schuler, An advanced culture method for generating large quantities of highly pure dendritic cells from mouse bone marrow, J. Immunol. Methods 223 (1999) 77-92.

19. P.M. Smith, M.R. Howitt, N. Panikov, M. Michaud, C.A. Gallini, Y.M. Bohlooly, J.N. Glickman, W.S. Garrett, The microbial metabolites, short chain fatty acids, regulate the colonic Treg cell homeostasis. Science 341 (2013) 569-573.

20. J.E. Koenig, A. Spor, N. Scalfone, A.D. Fricker, J. Stombaugh, R. Knight, L.T. Angenent, R.E. Ley, Succession of microbial consortia in the developing infant gut microbiome. Proc. Natl. Acad. Sci. USA 108 (2011) 4578-4585.

21. C.N. Manzotti, M.K. Liu, F. Burke, L. Dussably, Y. Zheng, D.M. Sansom, Integration of CD28 and CTLA-4 function results in differential responses of T cells to CD80 and CD86, Eur. J. Immunol. 36 (2006) 1413-1422.

22. H.R. Christensen, H. Frøkiær, J.J. Pestka. Lactobacilli Differentially Modulate Expression of Cytokines and Maturation Surface Markers in Murine Dendritic Cells.; J Immunol 168 (2002) 171 178.

23. S.G. Jeon, H. Kayama, Y. Ueda, T. Takahashi, T. Asahara, H. Tsuji, N.M. Tsuji, H. Kiyono, J.S. Ma, T. Kusu, R. Okumura, H. Hara, H. Yoshida, M. Yamamoto, K. Nomoto, K. Takeda, Probiotic Bifidobacterium breve induces IL-10-producing Tr1 cells in the colon, PLoS Pathog. 8(5) (2012) e1002714. 
24. G.D. Wu, J. Chen, C. Hoffmann, K. Bittinger, Y.Y. Chen, S.A. Keilbaugh, M. Bewtra, D. Knights, W.A. Walters, R. Knight, R. Sinha, E. Gilroy, K. Gupta, R. Baldassano, L. Nessel, H. Li, F.D. Bushman, J.D. Lewis, Linking long-term dietary patterns with gut microbial enterotypes. Science 334 (2011) 105-108. 


\section{Figure Legends}

Figure 1. FACS analysis of DCs from BALB/c mice. Immature DCs (iDCs) were challenged with irradiated lactobacilli (from subjects n. 3, 18 and 42 of Table 1) or bifidobacteria (from subjects n. 14, 21 and 39 of Table 1) and then subjected to a 6-h LPS pulse to induce DC maturation (mDCs). Both iDCs and treated or untreated mDCs were double stained for CD80 and CD86. Data were collected from ungated cells and are representative of three independent experiments.

Figure 2. Expression of the co-stimulatory markers CD80 and CD86 on the surface of mDCs. mDCs were pre-treated with irradiated lactobacilli (a) or bifidobacteria (b) isolates from adults who followed an omnivorous ( $\mathrm{n}=55,40$; a, b), ovo-lacto-vegetarian (n. 53, 36; a, b) or vegan (n. 47, 34; a, b) diet. Data were collected from ungated cells, and bars represent medians. * $P<0.05$; **, $P<$ 0.01 .

Figure 3. Setup of cytokine expression by DCs. a) Kinetic analysis of IL-12, IL-10 and TNF- $\alpha$ transcription in iDCs in response to LPS pulse; values were calculated as fold change in gene expression and expressed as \% of maximum induction. b) Comparison between mRNA and cognate protein levels for IL-12 (upper panel), IL-10 (middle panel) and TNF- $\alpha$ (bottom panel) of DCs pretreated with lactobacilli from subjects n. 3, 18 and 24 collected for three weeks. Cells and culture supernatants were collected as indicated in the Materials and Methods section and analysed for cytokine mRNAs and secreted protein expression by using real-time PCR and sandwich-type ELISA, respectively; values are expressed as \% of control (unchallenged mDCs); columns represent the mean $\pm \mathrm{SD}$ and are representative of three independent experiments. ${ }^{*}, P<0.05$.

Figure 4. Protein expression of IL-12 and IL-10 by mDCs. mDCs were pre-treated with irradiated lactobacilli (a, c) or bifidobacteria (b, d) isolates from adults who followed an omnivorous (n. 55, 40; a and c, b and d), ovo-lacto-vegetarian (n. 53, 36; a and c, b and d) or vegan (n. 47, 34; a and c, $\mathrm{b}$ and $\mathrm{d}$ ) diet before LPS pulse. Culture supernatants were collected after $24 \mathrm{~h}$ and analysed for IL$12(\mathrm{a}, \mathrm{b})$ and IL-10 (c, d) expression by sandwich-type ELISA. Data were expressed as \% of control (unchallenged mDCs); bars represent medians. **, $P<0.01$; ***, $P<0.001$.

Figure 5. Protein expression of TNF- $\alpha$ by mDCs. mDCs were pre-treated with irradiated lactobacilli (a) or bifidobacteria (b) isolates from adults who followed an omnivorous ( $\mathrm{n}=55,40$; a, b), ovo-lacto-vegetarian $(n=53,36$; a, b) or vegan $(n=47,34$;,$b)$ diet before LPS pulse. Culture 
supernatants were collected after $24 \mathrm{~h}$ and analysed by sandwich-type ELISA. Data were expressed as $\%$ of control (unchallenged mDCs); bars represent medians. ***, $P<0.001$.

Figure 6. Anti-inflammatory potential of diet-selected lactobacilli and bifidobacteria. Cytokine values reported in Fig. 4 were expressed as IL-10/IL-12 ratios for each lactobacilli and bifidobacteria population from adults who followed an omnivorous, ovo-lacto-vegetarian or vegan diet. Bars represent medians. ${ }^{*}, P<0.05$.

Figure 7. Anti-inflammatory potential of lactobacilli and bifidobacteria analysed across diet and geography. Cytokine values reported in Fig. 4 were expressed as IL-10/IL-12 ratios for lactobacilli and bifidobacteria populations from adults recruited at four Italian centres who followed an omnivorous, ovo-lacto-vegetarian or vegan diet (see Table 1). Bars represent medians. ${ }^{*}, P<0.05$; **, $P<0.01$. 
Table 1. List of mesophilic lactobacilli and bifidobacteria isolates from human faecal samples in this study.

\begin{tabular}{|c|c|c|c|c|}
\hline $\begin{array}{c}\text { Recruitment } \\
\text { Centre }\end{array}$ & Bacteria & & Diet Regimen & \\
\hline \multirow[b]{3}{*}{ Turin } & & Omnivorous $\left(\mathrm{n}^{*} ;\right.$ code $\left.^{* *}\right)$ & Vegetarian $\left(n^{*} ; \operatorname{code}^{* *}\right)$ & Vegan $\left(\mathrm{n}^{*} ;\right.$ code $\left.* *\right)$ \\
\hline & Lactobacilli & $15(1-15)$ & $12(16-24 ; 26-28)$ & $13(30-42)$ \\
\hline & Bifidobacteria & $15(1-15)$ & $13(16-23 ; 25-29)$ & $\begin{array}{c}11(30-33 ; 35 ; 36 ; 38- \\
42)\end{array}$ \\
\hline \multirow[b]{2}{*}{ Parma } & Lactobacilli & $10(43-52)$ & $15(53-67)$ & $12(68-79)$ \\
\hline & Bifidobacteria & $8(45-52)$ & $\begin{array}{c}11(53 ; 54 ; 56-60 ; 62 ; 65- \\
67)\end{array}$ & $9(68 ; 71 ; 73-79)$ \\
\hline \multirow[b]{2}{*}{ Bari } & Lactobacilli & $15(80-103)$ & $12(104-107 ; 109-116)$ & $10(120-129)$ \\
\hline & Bifidobacteria & $15(80-103)$ & $11(105-113 ; 115 ; 116)$ & $\begin{array}{c}12(118-125 ; 127- \\
129)\end{array}$ \\
\hline \multirow{2}{*}{ Bologna } & Lactobacilli & $15(130-144)$ & $14(145-158)$ & $12(159-170)$ \\
\hline & Bifidobacteria & $2(136 ; 139)$ & $1(146)$ & $2(161 ; 166)$ \\
\hline \multirow{2}{*}{ Total } & Lactobacilli & 55 & 53 & 47 \\
\hline & Bifidobacteria & 40 & 36 & 34 \\
\hline
\end{tabular}

*: n, number of subjects.

**: code number, arbitrarily assigned to identify subjects. 

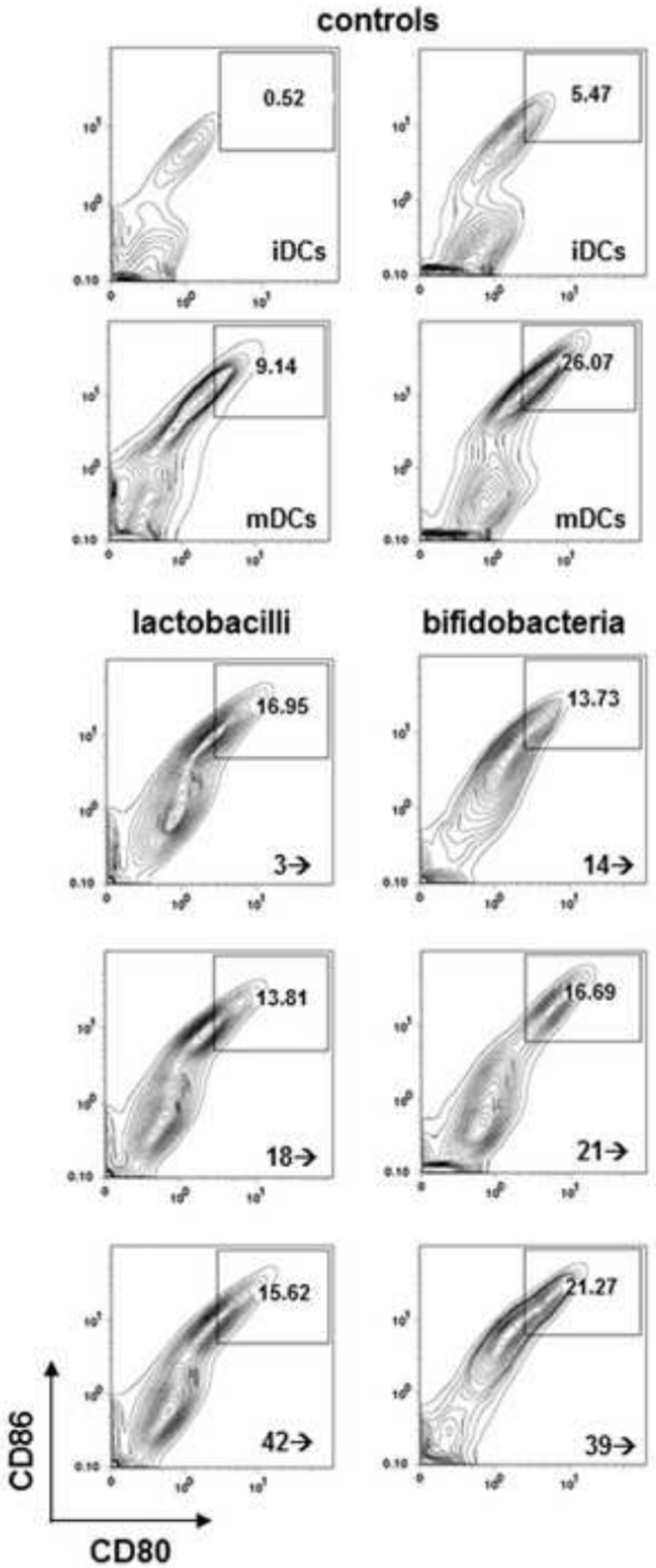
a

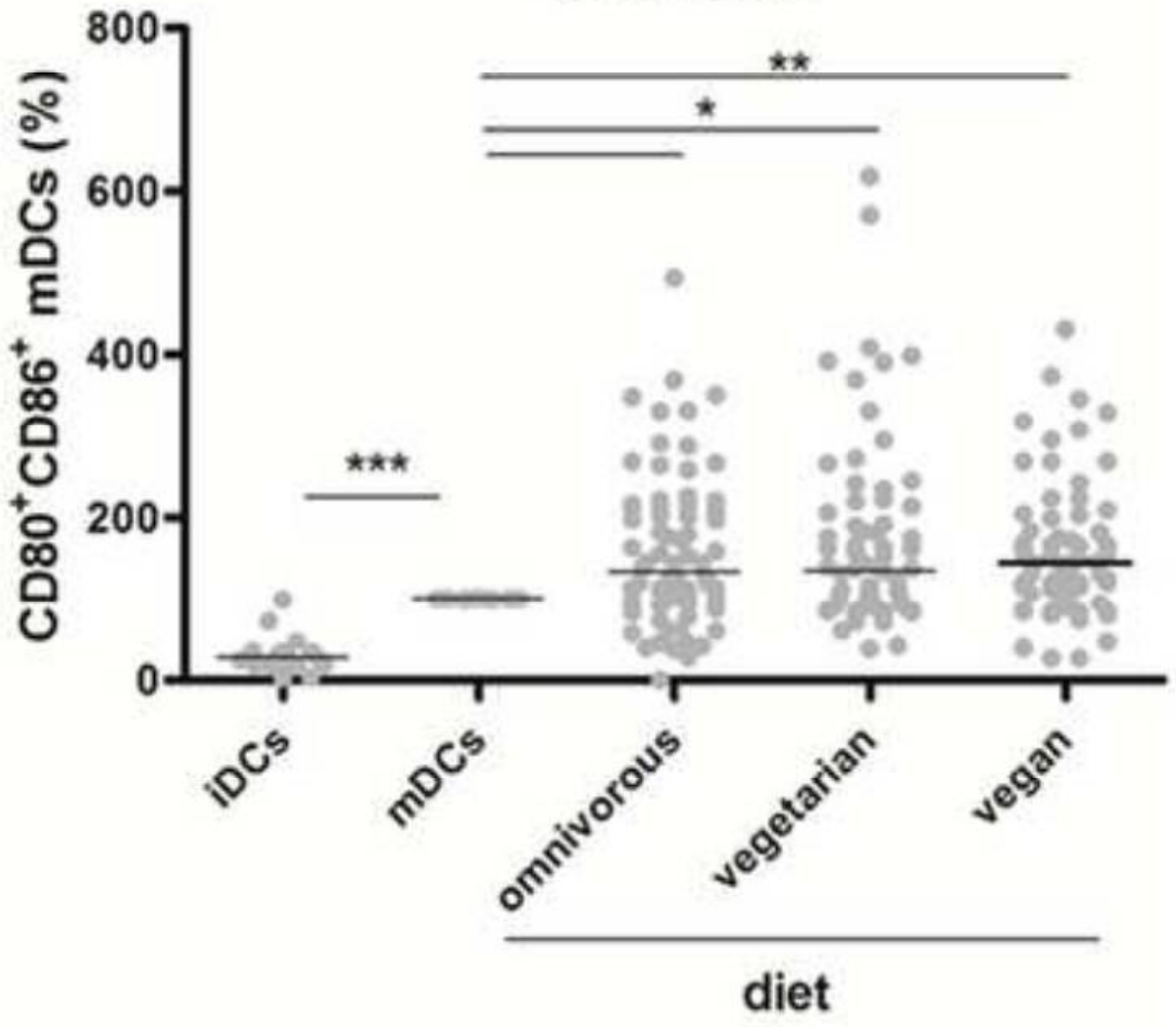

b

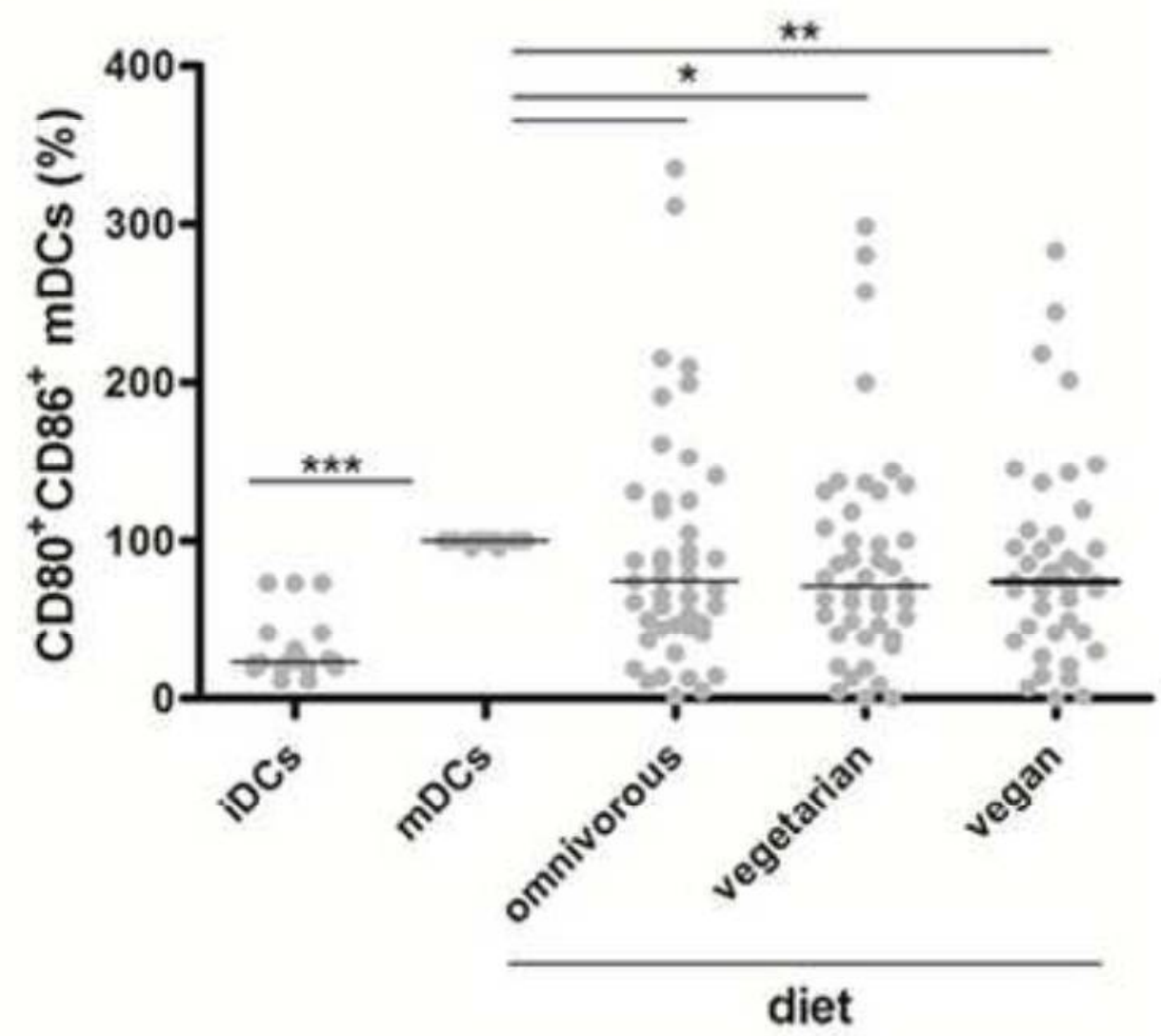




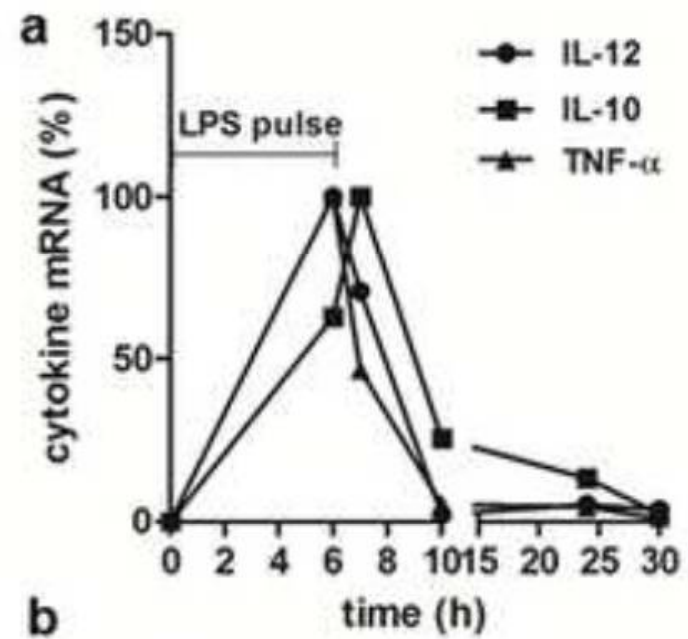

b
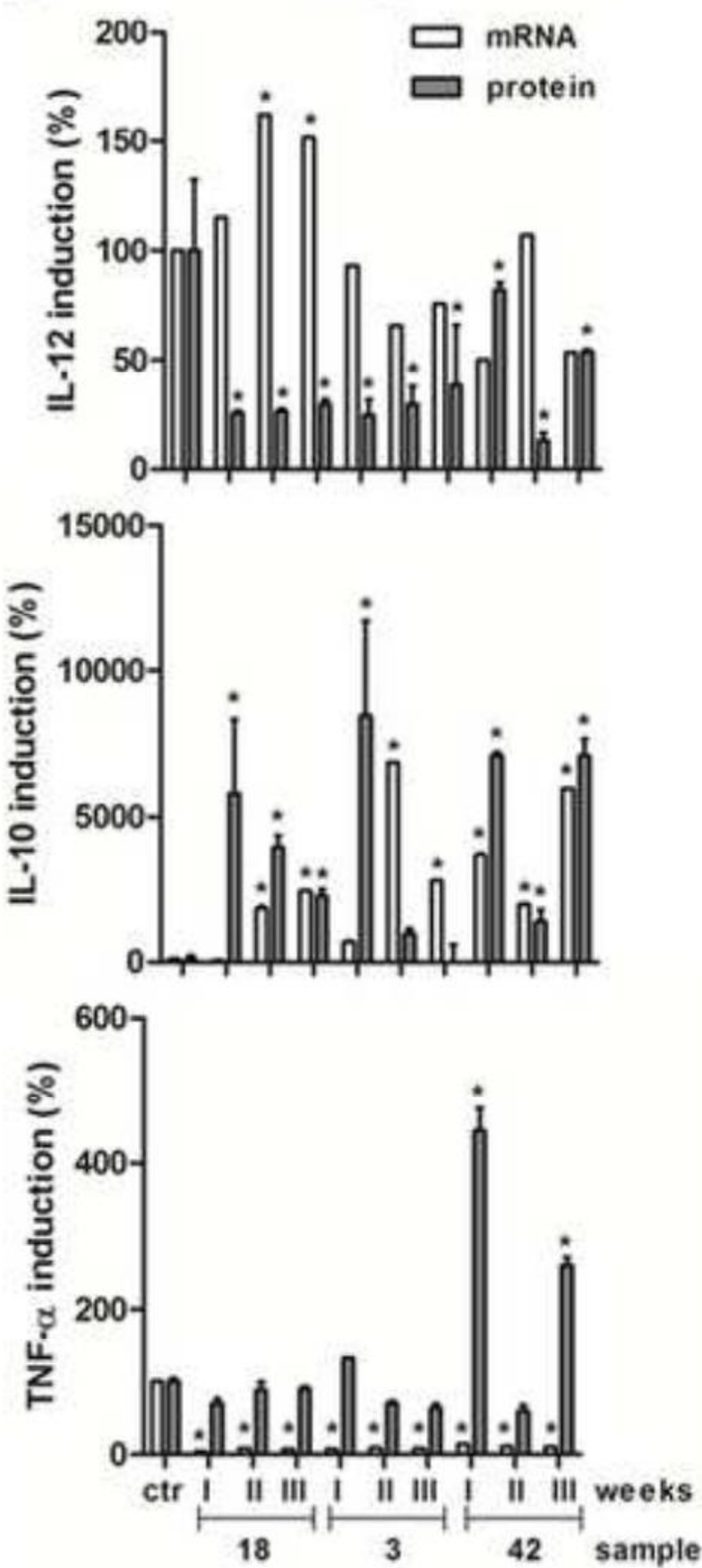
a

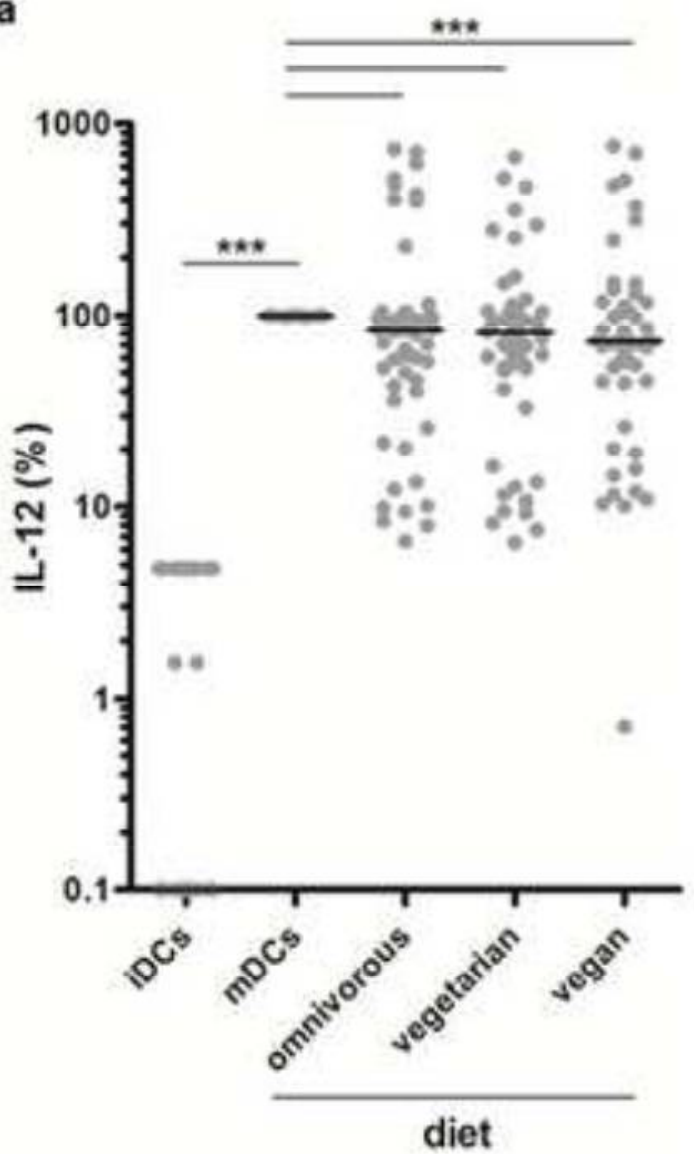

c

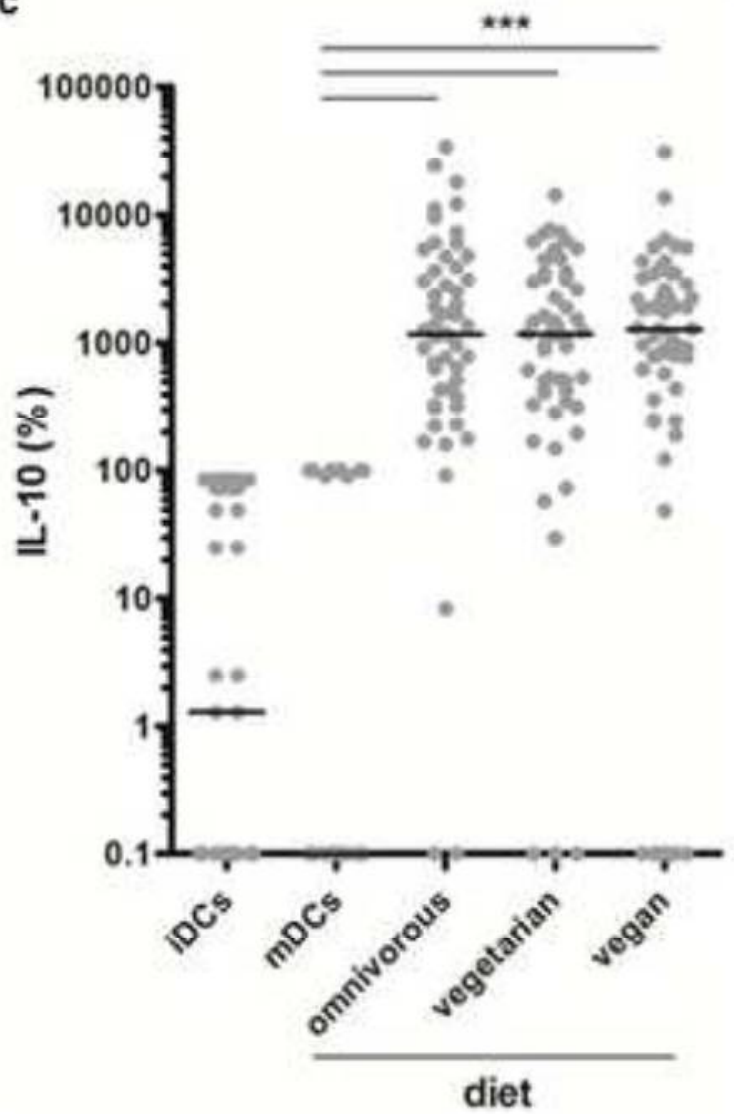

lactobacilli

b

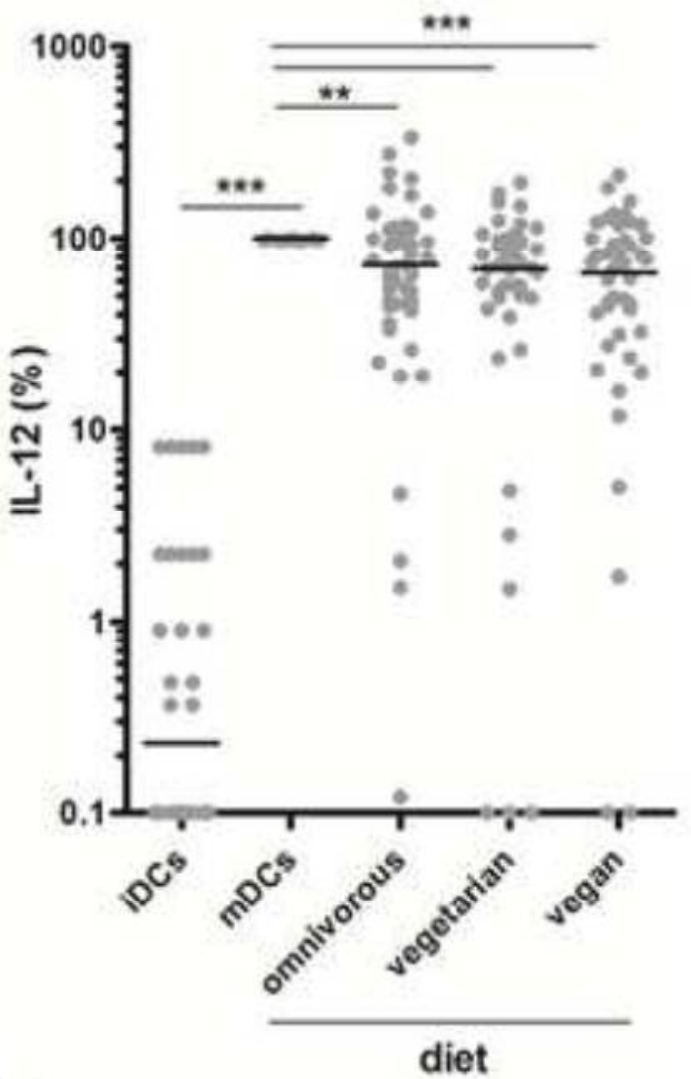

bifidobacteria

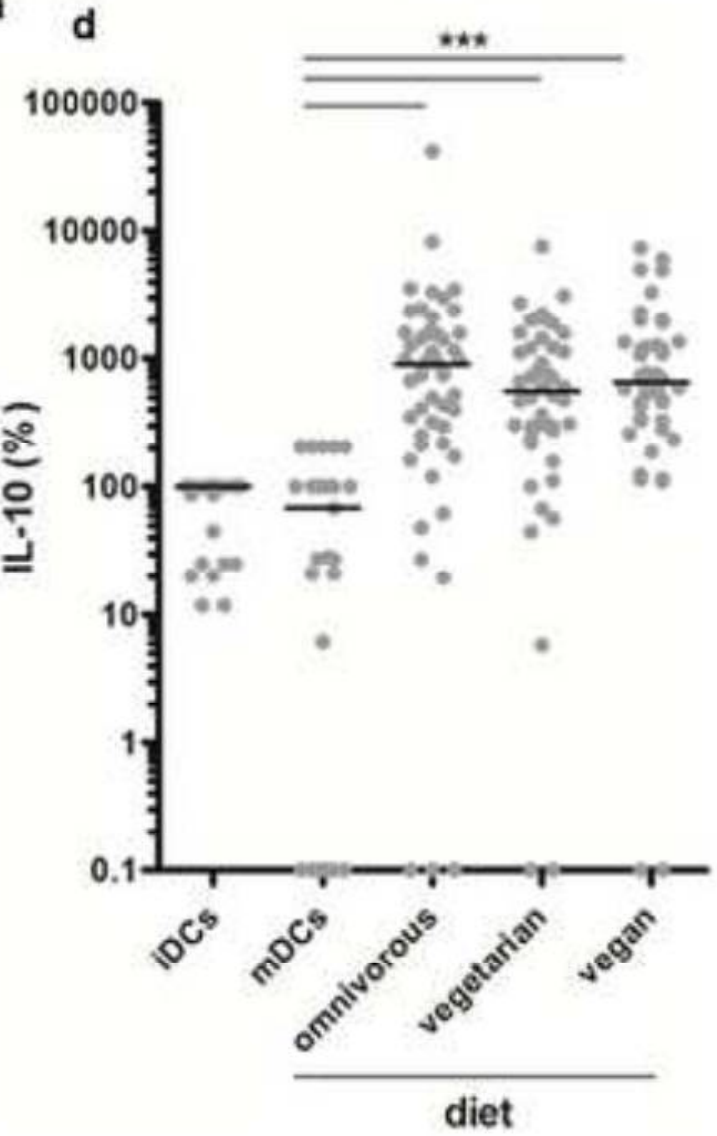


a

lactobacilli

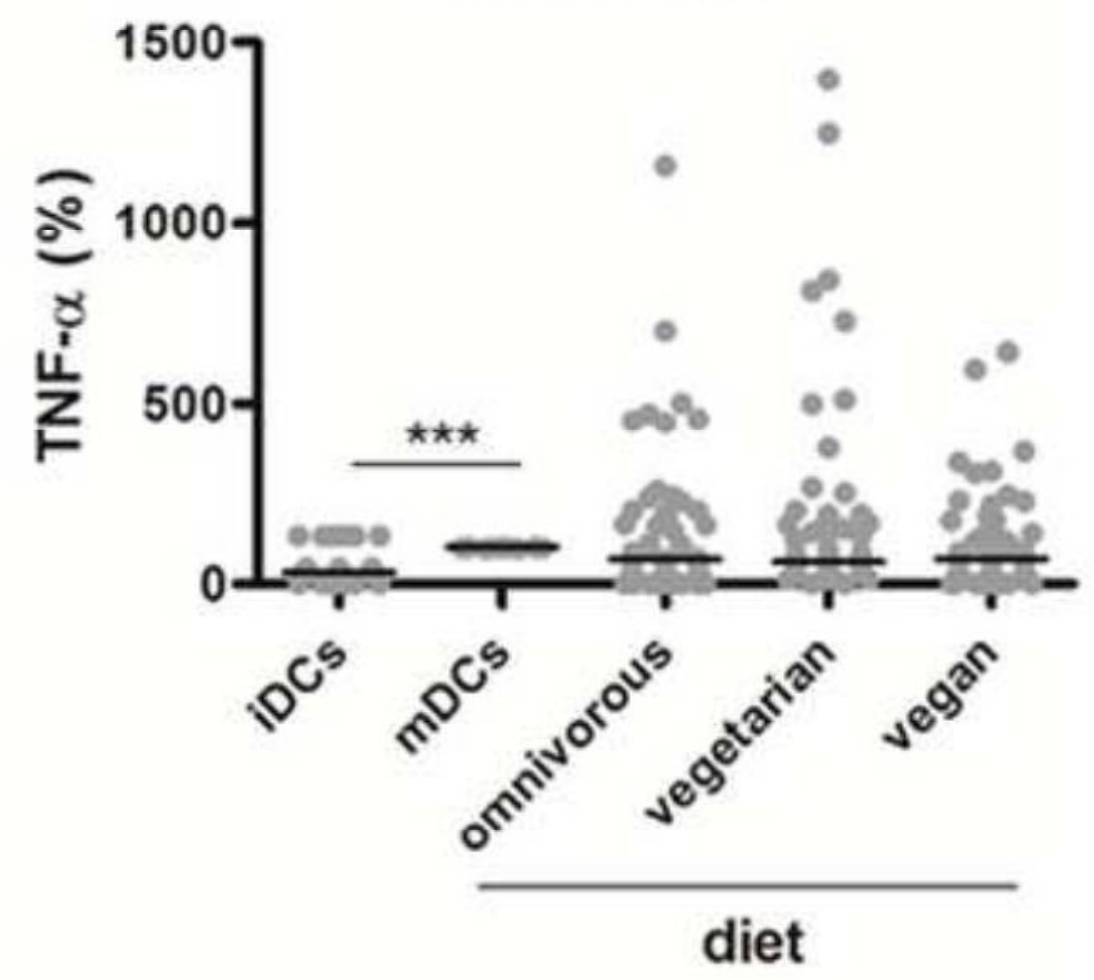

b

bifidobacteria

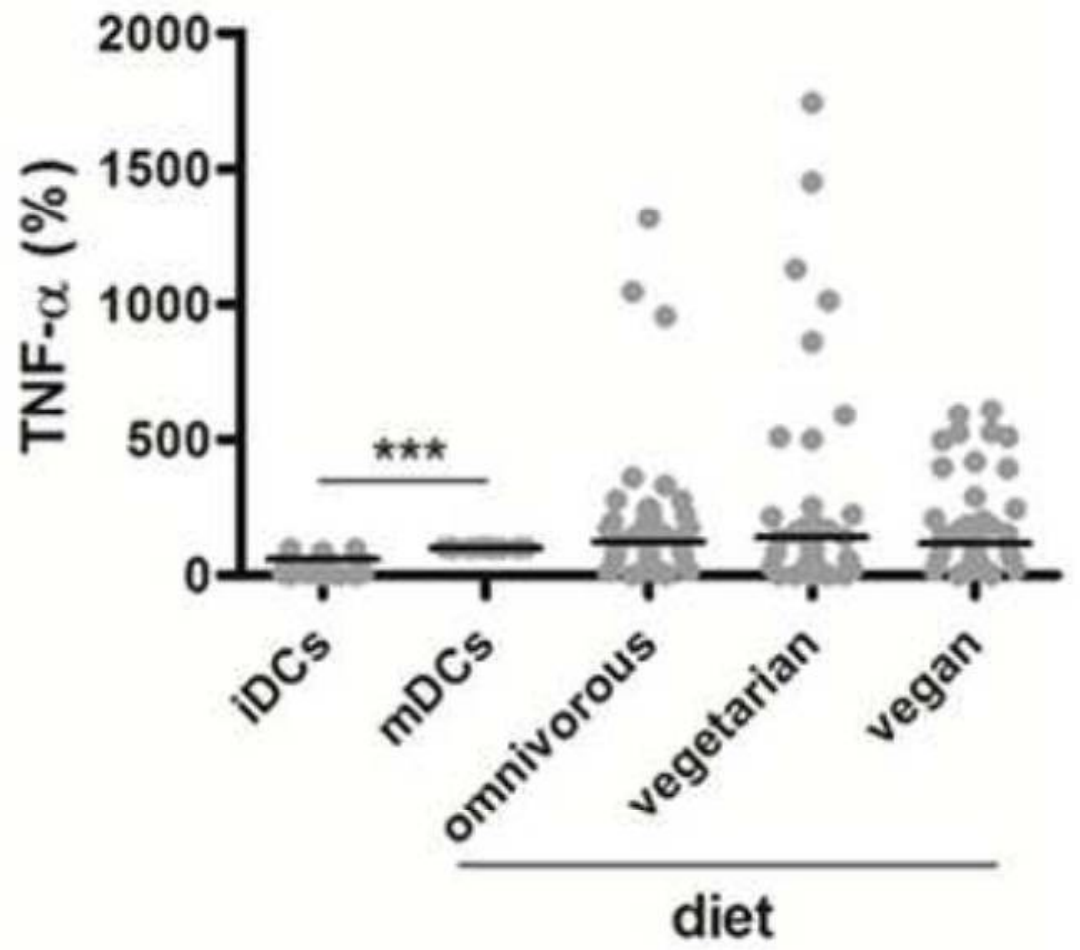




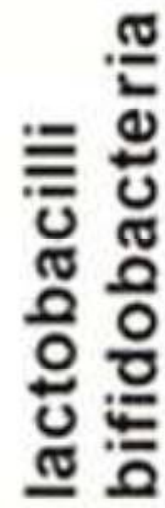

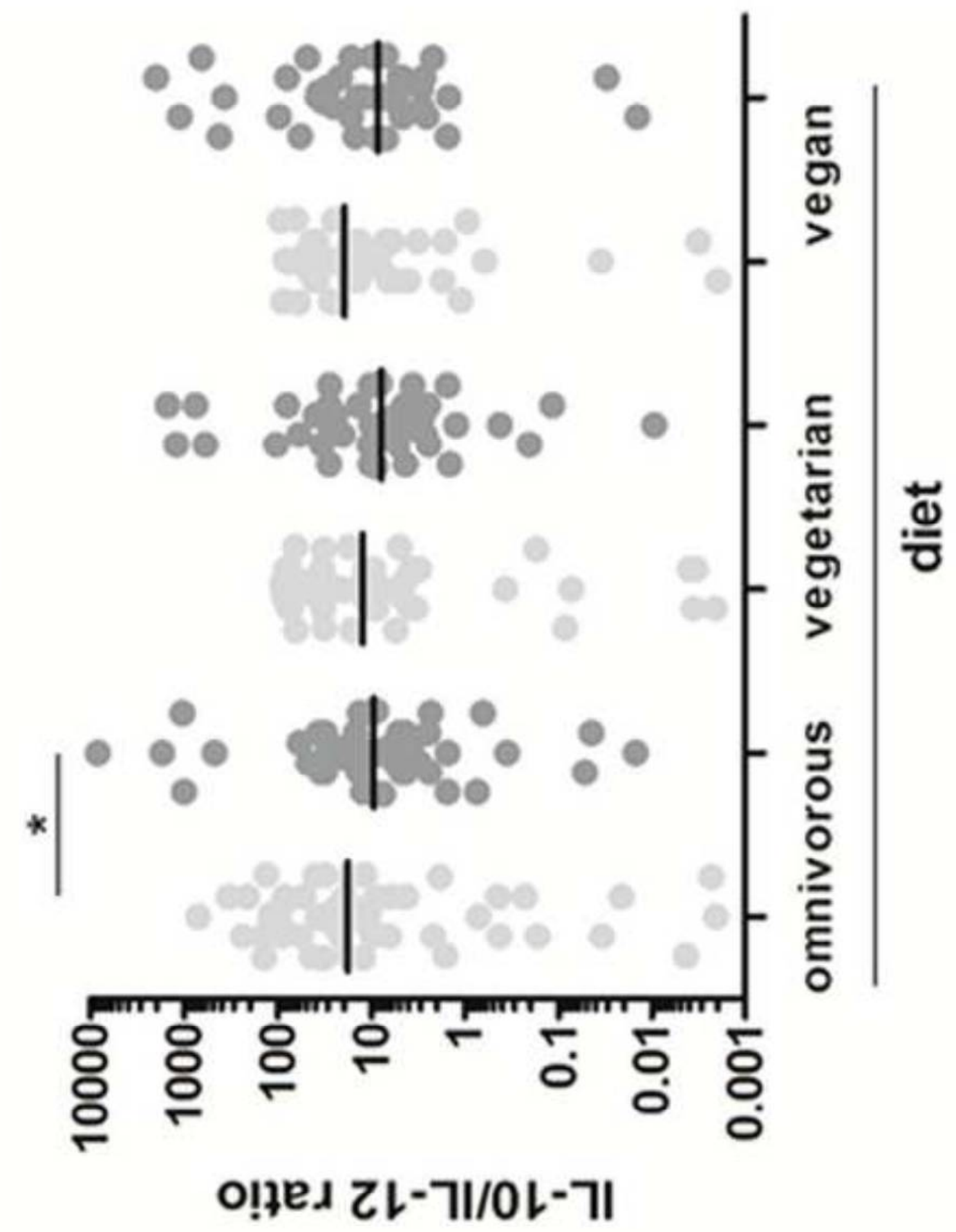

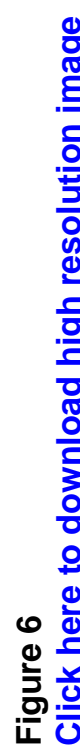


Click here to download high resolution image

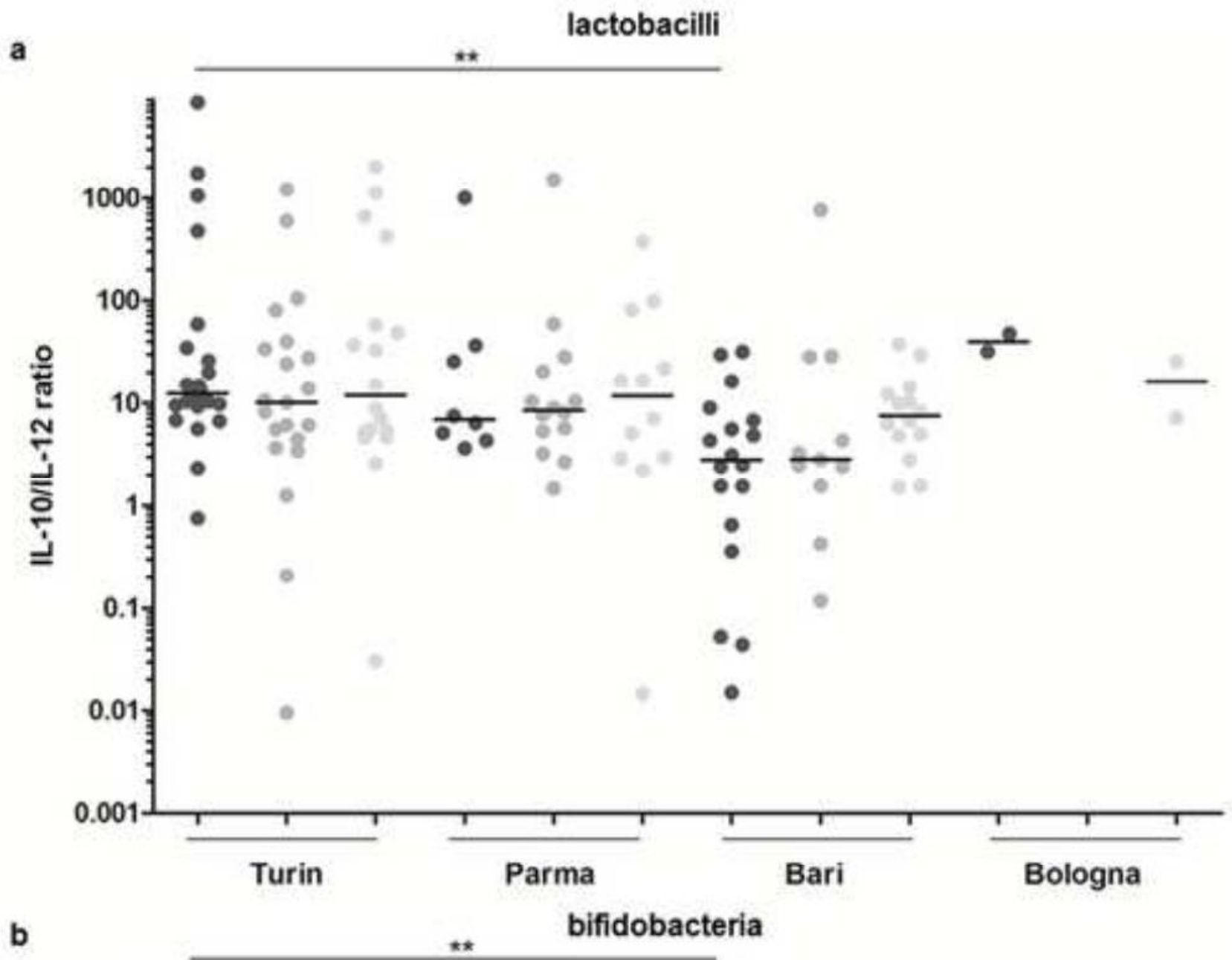

b

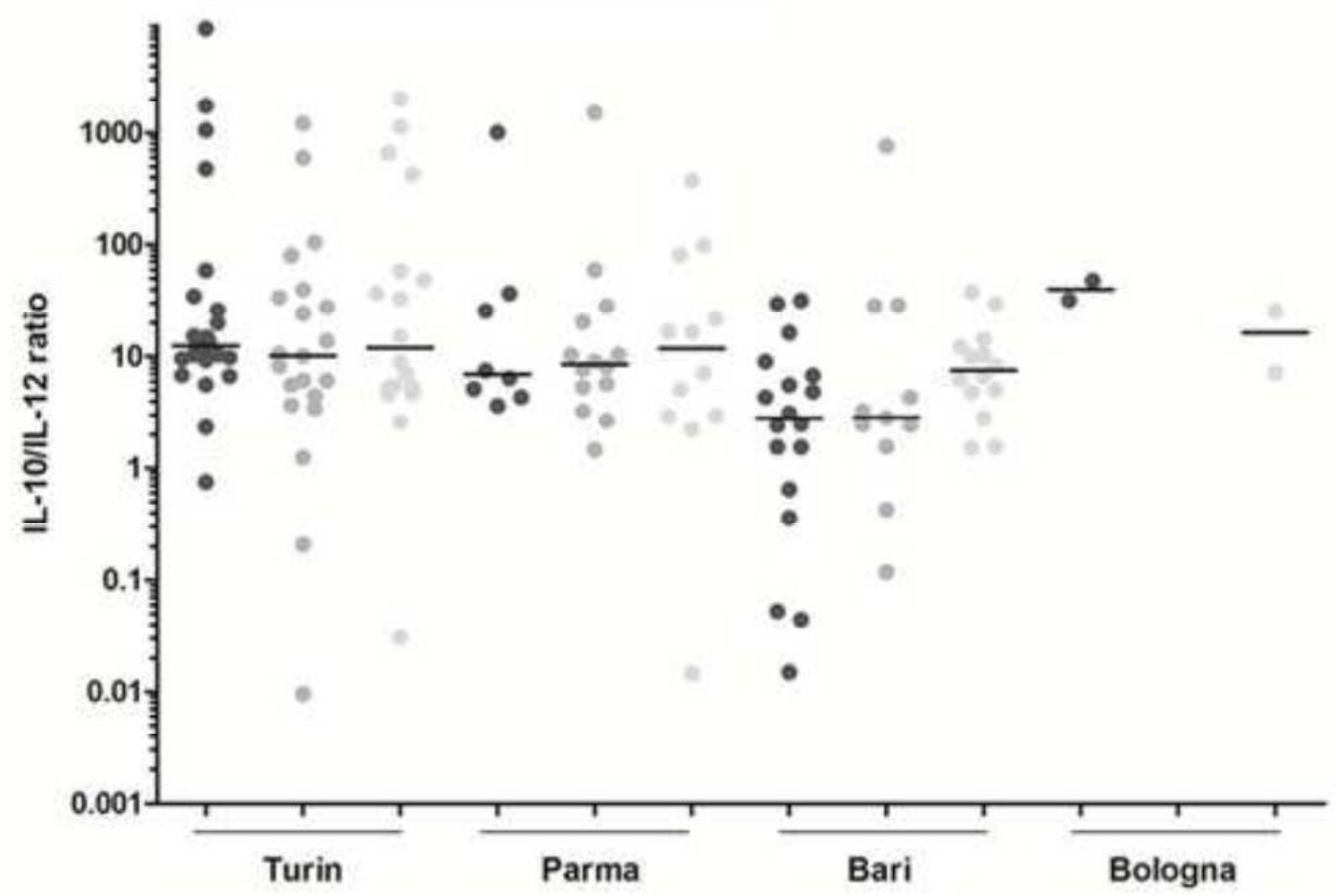

